\title{
Bi-orthogonal decomposition and Wavelet transform to analyse kinematics and dynamics of surface waves
}

\author{
by M. Houri*, \\ C. Kharif**, J.P. Giovanangeli**
}

\begin{abstract}
Summary
A row of discrete vortices is superposed to an air-sea interface to modelize the generation of surface waves by wind. A numerical method is developed in order to take into account the nonlinear effects which occur in the evolution of surface waves with finite steepness. The shape of the excited surface waves range from quasi sinusoidal to asymmetrical profiles close to breaking. Bi-orthogonal decomposition shows that resonant excitation (amplification of wave amplitude) may occur both at the fundamental frequency and at its second harmonic. This deterministic physical model is compared with experiments conducted in an air-sea tunnel. A vortex street is artificially created in the air flow. Using Fourier analysis and Wavelet transform it is found, in agreement with the numerical formulation, that non-sinusoidal disturbances advected over the water surface can have harmonic components that interact resonantly to generate surface waves, even thought the basic (fundamental) scale is non -resonant.
\end{abstract}

\section{Introduction}

In ocean, surface waves under the action of wind can lead to the formation of extreme sea-states characterized by the occurrence of abnormal waves, so called giant waves or freak waves. The giant waves particularly when they break, are dangerous to the navigation and to offshore structures. In this context, the meeting of air vortices and surface waves can give rise to breaking wave which in turn can destroy ships and tankers. The knowledge of the dynamic and kinematic properties of these extreme waves is very important from a practical point of view. Indeed it can help particularly, the engineers who are in charge of the design of vessels and marine structures.

$\mathrm{Lamb}^{1)}$ is one of the first authors who studied the response of a water surface subject to a moving pressure field propagating above it. His analysis is linear and showed that the wave amplitude is maximum when the resonance condition is satisfied, i. e. the propagation speed of the pressure field is equal to the surface wave speed. The Phillips' model $^{2)}$ introduces the same resonant interaction between surface waves and pressure fluctuations in turbulent boundary layer which is

* Ship Dynamics Division, Ship Research Institute

** I. R. P. H. E., laboratoire I. O. A., case 903, 13288, Marseille, France

Received 9th July 1997

Read at the Autumn meeting 14, 15th Nov. 1997 convected downstream above the interface. Phillips' model is also linear and describes fairly well the initial stage of waves generation in which the resonance process seems to be the main mechanism. The model proposed by Miles ${ }^{3)}$ rely on a different physical mechanism : the mean boundary layer velocity profile induces a pressure perturbation which is linear in the surface height, driving an instability with an exponential growth rate.

The Phillips' resonance mechanism is an uncoupled model in the sense that excitation is assumed to be independent of response. Miles' theory is a coupled model in the sense that excitation is assumed to be proportional to the surface height. $\mathrm{Miles}^{4)}$ in 1959, provided a model which synthesizes the two mechanisms.

The difficulties of the original models in describing the observed growth rates, motivated further developments in the theory of surface wave generation by wind. Unfortunately, the prediction of the energy transfer from wind to surface waves is governed by a nonlinear process which is difficult to deal with directly because of the free boundary and the inherent nonlinearity. Numerical computations have been developed to understand this sort of phenomenon.

In the last decade, different numerical formulations modeling the unsteady motion of a nonlinear free surface subject to an external perturbation have been developed. Since all of the interior properties of a body of fluid undergoing inviscid, incompressible irrotational motion are fully determined by properties at its boundary, it is possible to reduce the calculation of the motion 
of such a fluid to the evaluation of the motion of its surface alone. In a numerical scheme, the entire motion can thus be modeled using only a point discretisation of the surface. Such an approach was first suggested by Svendsen ${ }^{5}$. Since then, the idea has been implemented, using a variety of boundary integral methods (Longuet -Higgins and Cokelet $^{6)}$; Vinje and Brevig ${ }^{7)}$; Zarodny and Greenberg $^{8)}$; Baker et al. $^{9)}$ ).

Experimentally, the generation of resonant surface waves by discrete air vortices in experimental facilities is not so evident due to the complexity in detecting and measuring coherent structures in boundary layers. In order to overcome this difficulty, Giovanangeli and Memponteil ${ }^{10)}$, using an oscillating airfoil, created a vortex street convected by the wind above the interface. They observed experimentally the generation of waves when the Phillips resonance condition is satisfied: a vortex street of wavelength $\mathrm{L}$ generates surface waves of the same spatial scale (wavelength $\lambda=\mathrm{L}$ ) if it is moved with a uniform velocity, $U^{a}$, satisfying the dispersion relation of surface waves, $U^{a}=\mathrm{c}(\mathrm{k})$, where $\mathrm{c}(\mathrm{k})$ and $\mathrm{k}$ represent the fundamental wave phase speed and wave number respectively. They also suspected that waves may also be generated at higher resonances and use of adequate signal analyses tools should be of great interest.

In the papers cited above, external disturbances (vortices and the induced pressure perturbation) generate only the fundamental resonant wave harmonic with the same dynamics and kinematics properties $(\mathrm{c}(\mathrm{k})=$ $\left.U^{a}, \lambda=\mathrm{L}\right)$. Within the author's knowledge no work predicting the generation of resonant waves of spatiotemporal scales different from those of the vortex street has been published yet. In addition, use of new signal analyses such as bi-orthogonal decomposition (referred hereafter as B. O. D.) and Wavelet transform (referred hereafter as W. T.) seems to be of a crucial interest in analyzing such a type of generated surface waves. These methods are respectively adopted to observe time amplification of wave harmonics and sporadic phenomenon which occur very often and are undetected by the Fourier analysis. This challenging problem together with the extension of the B.O.D. and W. T. to the analysis of the kinematics and the dynamics properties of surface waves motivated our work.

The principal aims of the present study can be expressed very simply as follows: (i ) solve the exact windwave governing equations (ii) present experiments conducted in a wind-wave tunnel (iii) show the application of B. O. D. and W. T. in analyzing kinematics and dynamics of the generated numerical and experimental surface waves.

The paper is organized as follows. Section 2 presents briefly B. O. D. and W. T. and gives references for more details concerning these two methods. In $\S 3$, we construct the numerical method solving exact nonlinear wind-wave governing equations representing, as in Novikov ${ }^{11}$, coherent structures present in wind, as an array of point vortices flowing near the surface. Using B. O.D., we show the time amplification of wave's harmonic and specify the conditions for when the selective resonance mechanism, responsible for wave growth, occurs. In Section 4, Fourier analysis and W. T. are applied to the data obtained in a wind-wave tank experiment. A qualitative comparison between numerical and experimental models is presented. It is shown how W. T. can provide information undetected by Fourier analysis. Finally, section 5 gives a brief discussion of the results and outlines the crucial role of $B$. $O$. D. and W.T. in the selective resonance mechanism responsible for surface wave generation.

\section{Bi-orthogonal decomposition and Wavelet transform}

\subsection{Bi-orthogonal decomposition: a space-time decomposition}

B. O. D. is an analysis tool which provides a way to study in the space and time properties simultaneously. We present hereafter the most important features of the B. O. D. (for more details see Aubry et al. ${ }^{12)}$; Houri et al. $\left.{ }^{13), 14)}\right)$.

Suppose that our system is described by a function $\mathrm{u}(\mathrm{x}, \mathrm{t})$ defined on a spatial range $\mathrm{X}$ and a temporal interval $T$. The B. O. D. provides the smallest linear subspace $\chi(\mathrm{X})$ containing the phase space trajectory $\zeta_{t}$ (described as time $t$ runs) defined by

$\forall \mathrm{x} \in \mathrm{X}, \zeta_{\mathrm{t}}(\mathrm{x})=\mathrm{u}(\mathrm{x}, \mathrm{t})$

The set of all the vectors $\zeta_{\mathrm{t}}$ is the trajectory and the evolution of $\zeta_{t}$ is the dynamics of the system. The B. O. D. also provides the smallest linear subspace $\chi(\mathrm{T})$ containing the spatial structures $\zeta_{\mathrm{x}}$ (described as the spatial position $x$ varies) defined by

$\forall \mathrm{t} \in \mathrm{T}, \zeta_{\mathrm{x}}(\mathrm{t})=\mathrm{u}(\mathrm{x}, \mathrm{t})$.

In the present paper, the $\mathrm{L}^{2}$ scalar product is used to define $H(X)$ and $H(T)$, the Hilbert spaces of the functions of $\mathrm{x}$ on $\mathrm{X}$, and the functions of $\mathrm{t}$ on $\mathrm{T}$ respectively. The B. O. D. is the spectral analysis of the operator $U$, which acts from $\mathrm{H}(\mathrm{X})$ into $\mathrm{H}(\mathrm{T})$ :

$$
(\mathrm{U} \phi)(\mathrm{t})=\int \mathrm{u}(\mathrm{x}, \mathrm{t}) \phi(\mathrm{x}) \mathrm{dx}
$$

where $U$ defines a one-to-one relation between the vectors of $\chi(\mathrm{X})$ and $\chi(\mathrm{T})$, the orthogonal complements of the kernels of $U$ and its adjoint $U^{*}$. If $U$ is compact, the B. O. D. decomposes $u(x, t)$ into temporal and spatial orthogonal modes and $\mathrm{u}(\mathrm{x}, \mathrm{t})$ can be written as follows :

$$
\mathrm{u}(\mathrm{x}, \mathrm{t})=\sum_{\mathrm{n}=1}^{\mathrm{N}} \alpha_{\mathrm{n}} \psi_{\mathrm{n}}(\mathrm{t}) \phi_{\mathrm{n}}(\mathrm{x})
$$

with $\alpha_{1} \leq \alpha_{2} \geq \cdots \geq 0$, and the orthogonality relations ( $\phi_{n}$, $\left.\phi_{m}\right)=\delta_{n, m}\left(\psi_{n}, \psi_{m}\right)=\delta_{n, m}\left(\delta_{n, m}\right.$ is the Kronecker Symbol). The $\phi_{n}$ are called topos and the $\phi_{n}$ chronos. By analogy with the case for which the signal $u$ is a velocity field, we define the energy by

$$
E(u)=\int_{x} \int_{T} u(x, t) u(\bar{x}, t) d x d t=\sum_{n=1}^{\infty} \alpha_{n}^{2}
$$

From the above relation we see that each space-time structure, i. e. a pair $\left(\phi_{n}, \phi_{n}\right)$ participates independently 
to the energy of the signal. The normalized energy of each structure is :

$$
\mathrm{E}_{\mathrm{n}}=\frac{\alpha_{\mathrm{n}}^{2}}{\sum_{\mathrm{n}} \alpha_{\mathrm{n}}^{2}}
$$

and we define the global entropy of the signal by

$$
\mathrm{H}(\mathrm{u})=-\lim \frac{1}{\log N} \sum_{\mathrm{n}=1}^{\mathrm{N}} E_{\mathrm{n}} \log \mathrm{E}_{\mathrm{n}}, \quad \mathrm{N} \rightarrow \infty
$$

\section{2 Wavelet transform}

The wavelet transform is well documented. We present hereafter its basic properties. A good review of this method is given by Rioul and Duhamel ${ }^{15}$. The W. T. can be related to the more commonly used Fourier transform or Fourier series. The Fourier models represent functions as weighted sum of exponentials at different frequencies. The weight at each different frequency is the Fourier coefficients. Wavelet transform analogously models functions as a weighted sum of scaled and translated mother wavelets. The mother wavelet replaces the exponential, scaling and translation replace frequency shifting, and a two-dimensional surface of wavelet coefficient replaces the one dimensional Fourier coefficients. As proposed by Morlet et al. ${ }^{16)}$ the wavelet transform of a function $s(t)$ is the coefficients $\mathrm{T}_{\mathrm{s}}(\mathrm{b}, \mathrm{a})$ given by

$$
\mathrm{T}_{\mathrm{s}}(\mathrm{b}, \mathrm{a})=\sqrt{\mathrm{a}} \int_{-\infty}^{+\infty} \widehat{\mathrm{s}}(\omega) \hat{\psi}^{*}(\mathrm{a} \omega) \mathrm{e}^{\mathrm{ib} \omega} \mathrm{d} \omega
$$

where subscripts ${ }^{*}$ and - denote complex conjugation and Fourier transform respectively. The scale parameter ' $a$ ' and the translation parameter ' $b$ ' can be chosen to vary either continuously $(\mathrm{a}, \mathrm{b} \in \mathscr{R}$, with $\mathrm{a} \neq 0$ ) or in discrete way $\left(\mathrm{a}=\mathrm{a}_{0}^{m}, \mathrm{~b}=\mathrm{nb}_{0} \mathrm{a}_{0}^{m}, \mathrm{a}_{0}>1, \mathrm{~b}_{0}>0, \mathrm{~m}, \mathrm{n}\right.$ are integers). The mother (basic) wavelet $\psi$ must verify the admissibility condition

$$
\int_{-\infty}^{+\infty}\left|\frac{\bar{\psi}(\omega)}{\omega}\right|^{2} \mathrm{~d} \omega=A<+\infty
$$

The main motivation for the admissibility condition is that it implies the conservation of energy

$$
\frac{1}{\mathrm{~A}} \iint_{\mathscr{R}^{2}}\left|\mathrm{~T}_{\mathrm{s}}(\mathrm{b}, \mathrm{a})\right|^{2} \frac{\mathrm{dadb}}{\mathrm{a}^{2}}=\int_{-\infty}^{+\infty}|\mathrm{s}(\mathrm{t})|^{2} \mathrm{dt}
$$

and a reconstruction relation

$$
\begin{aligned}
& \mathrm{s}(\mathrm{t})=\frac{1}{\mathrm{~A}} \iint_{\mathscr{R}^{2}} \mathrm{~T}_{\mathrm{s}}(\mathrm{b}, \mathrm{a}) \psi_{\mathrm{ab}}(\mathrm{t}) \frac{\mathrm{dadb}}{\mathrm{a}^{2}} \\
& \text { where } \quad \psi_{\mathrm{ab}}(\mathrm{t})=\psi\left(\frac{\mathrm{t}-\mathrm{b}}{\mathrm{a}}\right)
\end{aligned}
$$

In this paper, a FFT based discrete wavelet transform algorithm is developed using Fourier transform of the Morlet basic wavelet

$$
\widehat{\psi}(\omega)=\pi^{-\frac{1}{4}}\left(\mathrm{e}^{-(\omega-\lambda)^{2} / 2}-\mathrm{e}^{\lambda^{2} / 2} \mathrm{e}^{-\omega^{2} / 2}\right)
$$

where $\lambda=\pi^{1 / 2}(2 / \ln 2)^{-1 / 2}$ and $e^{-\lambda^{2} / 2}$ is to make $\psi$ verifying the admissibility condition. This is performed by computing the inverse FFT (IFFT) of the convolution product of FFT's of the signal (surface wave elevation) and Morlet basic wavelet.

\section{Nonlinear computation of excited surface waves}

\section{1 Numerical formulation}

We consider two incompressible and inviscid fluids, air and water, with constant densities $\rho^{\mathrm{a}, \mathrm{w}}$ (superscripts $\mathrm{a}$ and $\mathrm{w}$ indicate air and water respectively). Wind waves are commonly believed to be very close to potential flows. Assuming this, the fluid velocities should then be of the form $\nabla \phi^{\mathrm{a}, \mathrm{w}}$, the gradient of scalar potentials $\phi^{\mathrm{a}, \mathrm{w}}$. For irrotational flow the momentum equation can be integrated both in air and water to give the Bernoulli equation along the interface

$$
\begin{aligned}
& \phi_{\mathrm{t}}^{\mathrm{a}}+\frac{1}{2}\left|\nabla \phi^{\mathrm{a}}\right|^{2}+\mathrm{g} \eta+\frac{\mathrm{p}^{\mathrm{a}}}{\rho^{\mathrm{a}}}=0 \\
& \phi_{\mathrm{t}}^{\mathrm{w}}+\frac{1}{2}\left|\nabla \phi^{\mathrm{w}}\right|^{2}+\mathrm{g} \eta+\frac{\mathrm{p}^{\mathrm{w}}}{\rho^{\mathrm{w}}}=0
\end{aligned}
$$

where $\mathrm{p}^{\mathrm{a}, \mathrm{w}}$ are the pressure in air and in water respectively. The interface equation is $z=\eta(\mathrm{x}, \mathrm{t})$, where $\mathrm{x}$ and $t$ are respectively horizontal coordinate and time. Gravity acceleration is denoted by g. Kinematic conditions, both in air and water, at the interface are:

$$
\eta_{\mathrm{t}}=\phi_{z}^{\mathrm{a}, \mathrm{w}}-\phi_{x}^{\mathrm{a}, \mathrm{w}} \eta_{\mathrm{x}}
$$

Velocity potentials of air and water satisfy Laplace equation with conditions at infinity

$\Delta \phi^{\mathrm{a}, \mathrm{w}}=0 \quad \lim \phi^{\mathrm{a}, \mathrm{w}}=0 \quad z \rightarrow \pm \infty$

Surface tension is neglected so that pressure is continuous throughout the interface

$$
\mathrm{p}^{\mathrm{a}}=\mathrm{p}^{\mathrm{w}}=\mathrm{p}(\mathrm{x}, \mathrm{t})
$$

For the more general case of two different fluids separated by an interface with surface tension (gravity capillary wave), a similar but more complicated nonlinear equations can be derived. The pressure at the interface will have a discontinuity proportional to the curvature. Consideration of surface tension effects will be the aim of a future work

The set of the above equations governs the nonlinear free surface motion. The boundary integral method developed by Baker et al. ${ }^{9)}$ is used to solve the exact equations. In this paper, we present briefly this formulation and refer the readers for more details to the following references (Houri ${ }^{17), 18)}$, Baker et al. $\left.{ }^{9}\right)$. From classical potential theory, the interface is represented by a vortex sheet distribution of intensity $\mathbf{G}(\mathbf{x}, \mathbf{t})$ (see Fig. 1) due to a jump in tangential velocity. The vector $\boldsymbol{q}$ represents the velocity of "the free surface vortices", $\mathbf{N}$, $\mathbf{T}$ its normal and tangential components and $\mathbf{U}, \mathbf{V}$ its longitudinal and vertical components in a fixed frame of reference $(\mathbf{x}, \mathbf{z})$. Bernoulli equations (2) and ( 3 ) are first written in terms of our principal variables $\widetilde{\mathbf{G}}=$ $\mathbf{G} / \sqrt{1+\boldsymbol{\eta}_{x}^{2}}, \boldsymbol{\eta}, \mathbf{U}$ and $\mathbf{V}$ as follows :

$$
\begin{aligned}
& \phi_{\mathrm{t}}^{\mathrm{a}}+\frac{1}{2}\left[\left(\mathbf{T}-\frac{\tilde{\mathbf{G}}}{2}\right)^{2}+\mathbf{N}^{2}\right]+\mathbf{g} \eta+\frac{\mathrm{p}^{\mathrm{a}}}{\rho^{\mathrm{a}}}=0 \\
& \phi_{\mathrm{t}}^{\mathrm{w}}+\frac{1}{2}\left[\left(\mathbf{T}+\frac{\widetilde{\mathbf{G}}}{2}\right)^{2}+\mathbf{N}^{2}\right]+\mathbf{g} \eta+\frac{\mathrm{p}^{\mathrm{w}}}{\rho^{\mathrm{w}}}=0
\end{aligned}
$$

Next, using equation (5) and after some algebraic calculations, we obtain (for details see Houri ${ }^{17)}$; Zarodny and Greenberg ${ }^{8)}$ ) the following governing equation 


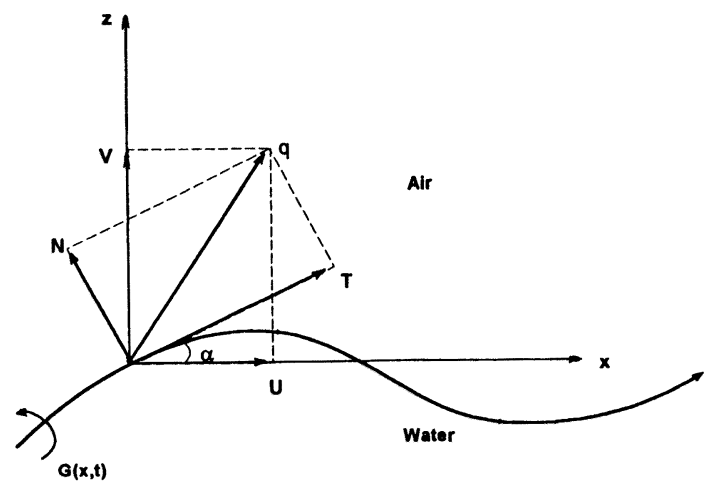

Fig. 1 The air-water interface representation

$$
\begin{aligned}
& \text { of } \mathrm{G}(\mathrm{x}, \mathrm{t}) \\
& \qquad \begin{aligned}
\frac{\mathrm{dG}}{\mathrm{dt}}= & -\mathrm{U}_{\mathrm{x}} \mathrm{G}+\frac{\kappa}{2}\left[\mathrm{C}^{2} \mathrm{G}\left(\mathrm{CSG} \eta_{\mathrm{xx}}-\mathrm{G}_{\mathrm{x}}\right)\right. \\
& \left.-4 \frac{\mathrm{dU}}{\mathrm{dt}}-4 \eta_{\mathrm{x}} \frac{\mathrm{dV}}{\mathrm{dt}}-4 \mathrm{~g} \eta_{\mathrm{x}}\right]
\end{aligned}
\end{aligned}
$$

where $\kappa=\left(\rho^{\mathrm{w}}-\rho^{\mathrm{a}}\right) /\left(\rho^{\mathrm{w}}+\rho^{\mathrm{a}}\right), \mathrm{C}=1 / \sqrt{1+\eta_{\mathrm{x}}^{2}}, \mathrm{~S}=\mathrm{C} \eta_{\mathrm{x}}$ and $\mathrm{d} / \mathrm{dt}$ represents a material derivative. Herein $\mathrm{U}$ and $\mathrm{V}$ may be regarded as the components of the velocity of "interface vortices" and are obtained using Biot-Savart relations (Houri $\left.{ }^{17)}\right)$. We also choose $\mathrm{L}=2 \pi$ and $\mathrm{g}=1$. Note that equation ( 6 ) is equivalent to equation (12) in Zarodny and Greenberg ${ }^{8)}$. Equation (6) and the kinematic conditions (4) together with given initial conditions $\eta(\mathrm{x}, 0)$ and $\mathrm{G}(\mathrm{x}, 0)$ are the main equations to be solved. The integration is accomplished using Adams Moulton method held in the supercomputer Cray ymp. The $\mathbf{N}$ points on the discretised surface used range from 100 points for initial wave steepness $\mathbf{a k}=0.1$ to 300 points for initial wave steepness $\mathbf{a k}=0.3$. The time step is controlled through the error parameter which is fixed to $10^{-4}$.

\section{2 Surface wave generation}

Since the reliability of the iterative vortex method has been demonstrated in Houri ${ }^{17)}$, we focus our attention on the application of the B. O. D. to surface wave generated by an external pressure distribution of the form

$$
\mathrm{p}^{(\Gamma)}=-\rho_{a}\left[\phi_{\mathrm{t}}^{(\Gamma)}+1 / 2\left|\nabla \varphi^{(\Gamma)}\right|^{2}\right]=\sum_{n=0} \mathrm{p}_{n} \mathrm{e}^{\mathrm{ink}\left(\mathrm{x}-\mathrm{U} a_{\mathrm{t}}\right)}
$$

( $n$ is an integer)

in which, we first consider that the Fourier coefficients $\mathrm{p}_{\mathrm{n}}\left(\right.$ for $\mathrm{n}>1$ ) are negligible. The velocity potential $\varphi^{(\Gamma)}$ is generated in air by a vortex street of intensity $\boldsymbol{\Gamma}$ and uniform velocity $U^{\mathrm{a}}$. Two successive vortices, horizontally aligned at height $\mathbf{H}$ from the surface level of water initially at rest, are separated by a distant $\mathbf{L}$. Since the vortex field is concentrated at the discrete vortices, fluid motion outside the vortices is potential, and the whole vortex street advances with a uniform velocity

$$
U^{\mathrm{a}}=\frac{\Gamma}{2 \mathrm{~L}} \operatorname{coth}(\pi \mathrm{H} / \mathrm{L}) .
$$

As it is seen from Fig. 2, the induced vortex pressure
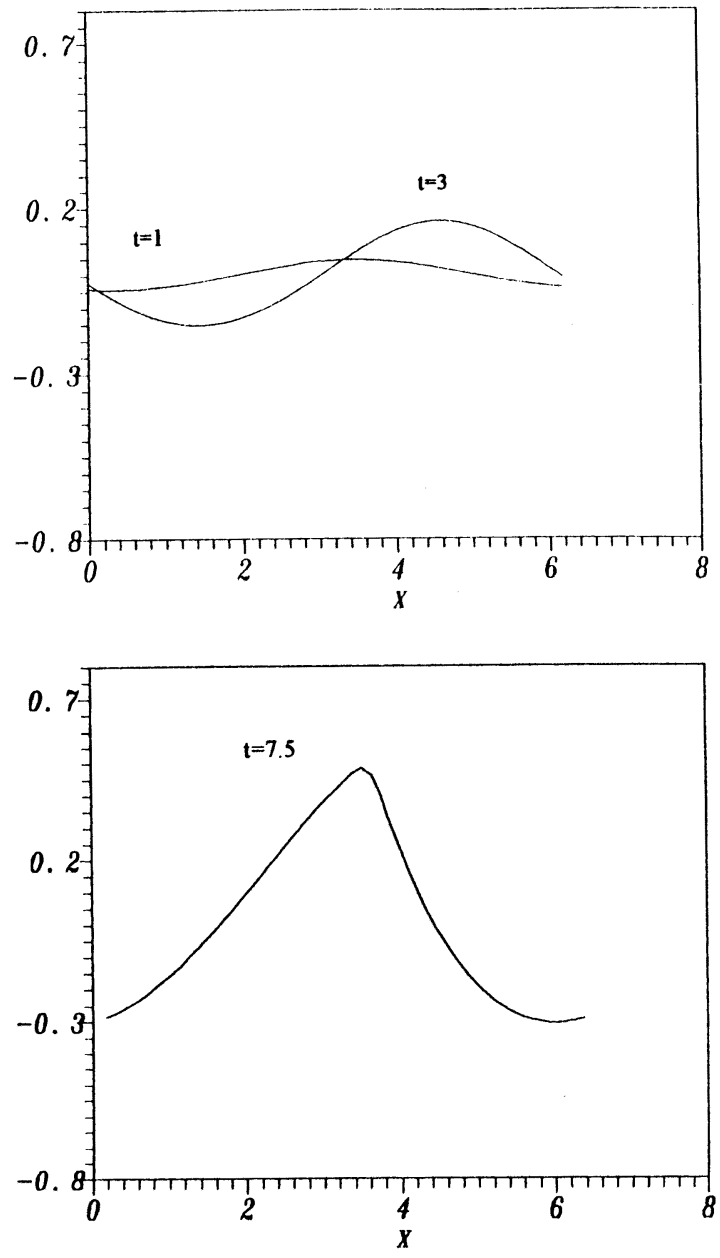

Fig. 2 Surface wave elevation $\left(\mathbf{p}_{1}=100\right)$ at various times

generates waves of growing amplitude in time which become asymmetric $(t=7.5)$, characteristic of highly nonlinear surface waves very close to breaking ones. Surface waves continue to grow in time until the curvature at the tip becomes too great to resolve with the number of points used.

Now, let the vortex street moving at the phase speed of the fundamental surface wave harmonic $\left(\mathrm{U}^{\mathrm{a}}=\mathrm{c}(\mathrm{k})=\right.$ $\sqrt{\mathrm{g} / \mathrm{k}}, \mathrm{k}=2 \pi / \mathrm{L}$ ) and let us apply B. O. D. to the generated waves in order to see their space and time properties simultaneously. Fig. 3 (a, b) displays the two first temporal modes (chronos) and the two first spatial modes (topos) of the surface elevation $\eta$. It is clearly shown that under the above condition $\left(\mathrm{U}^{\mathrm{a}}=\mathrm{c}(\mathrm{k})\right)$, the fundamental wave harmonic is resonantly generated characterized by a growing temporal modes (see the behavior of the chronos) and by spatial modes (topos) of wavelength equal to the distance separating two successive air vortices $(\mathbf{L}=2 \pi)$. 

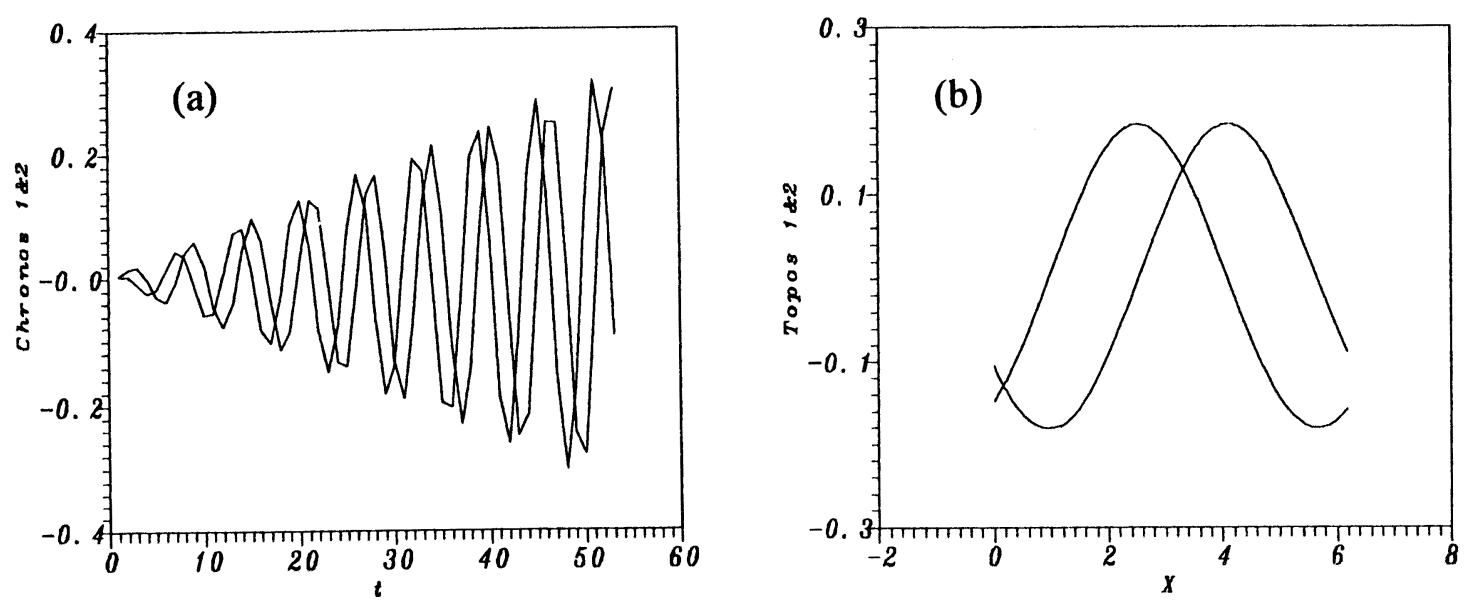

Fig. 3 Orthogonal temporal modes $\psi_{1} \& \psi_{2}$ (a) and orthogonal spatial modes $\phi_{1} \& \phi_{2}(\mathrm{~b})$ of the surface elevation $\left(\mathbf{p}_{1}=10\right)$
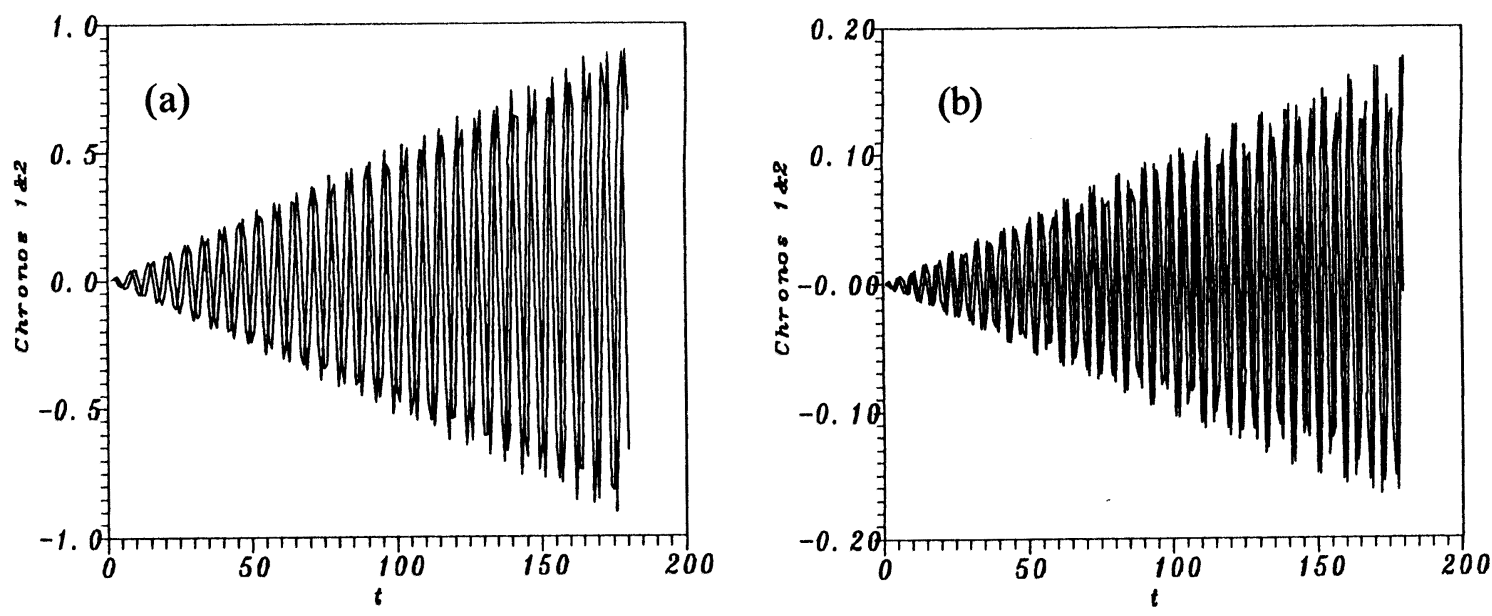

Fig. 4 Orthogonal temporal modes of the surface height $\psi_{1} \& \psi_{2}$ (a) $\mathbf{U}^{\mathbf{a}}=\mathbf{c}(\mathbf{k})$ and (b) $\mathbf{U}^{\mathrm{a}}=\mathbf{c}(\mathbf{k}) / \sqrt{2}$
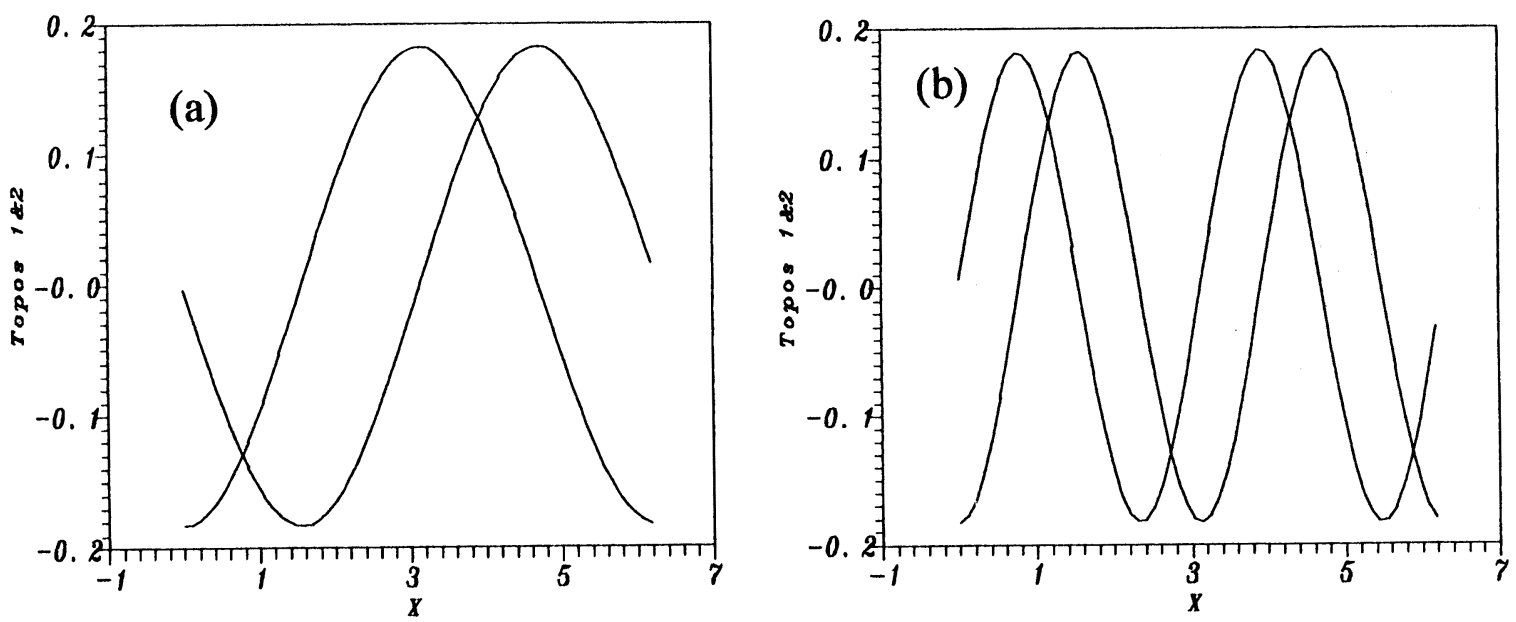

Fig. 5 Orthogonal spatial modes of the surface height $\phi_{1} \& \phi_{2}$ (a) $\mathbf{U}^{\mathrm{a}}=\mathbf{c}(\mathbf{k})$ and ( b ) $\mathbf{U}^{\mathrm{a}}=\mathbf{c}(\mathbf{k}) / \sqrt{2}$ 
Next, surface waves are generated by a periodic external pressure distribution with a two band spectrum $\left(\mathrm{p}_{1}\right.$ and $\mathrm{p}_{2}$ in Fourier distribution of $\left.\mathrm{p}^{(\Gamma)}\right)$. The vortex street is advected at two convection speeds: ( i ) at the fundamental wave phase speed $\left(\mathrm{U}^{\mathrm{a}}=\mathrm{c}(\mathrm{k})\right)$ and (ii) at the phase speed of the second harmonic $\left(\mathrm{U}^{\mathrm{a}}=\mathrm{c}(2 \mathrm{k})=\right.$ $\mathrm{c}(\mathrm{k}) / \sqrt{2})$. The B. O. D. applied both in cases ( i ) and (ii) for non-dimensionnal values of the circulation $\boldsymbol{\Gamma}=$ 1.3 and the height of the vortex street $\mathbf{H}=0.805$, shows that temporal modes (chronos) are growing in time as provided in Fig. $4(\mathrm{a}, \mathrm{b})$. This means that surface waves in both cases are generated and may break as time runs. But, it is not clear from these chronos what wave's harmonic (fundamental, superharmonic or subharmonic) is effectively being generated. From the orthogonal spatial modes plotted in Fig. $5(\mathrm{a}, \mathrm{b})$, it is shown that the wavelength of the generated surface wave is $\mathbf{L}$ for case ( i ) and $\mathbf{L} / 2$ for case (ii). Therefore, we can say that in the first case the fundamental wave harmonic is growing and in the second case the superhar
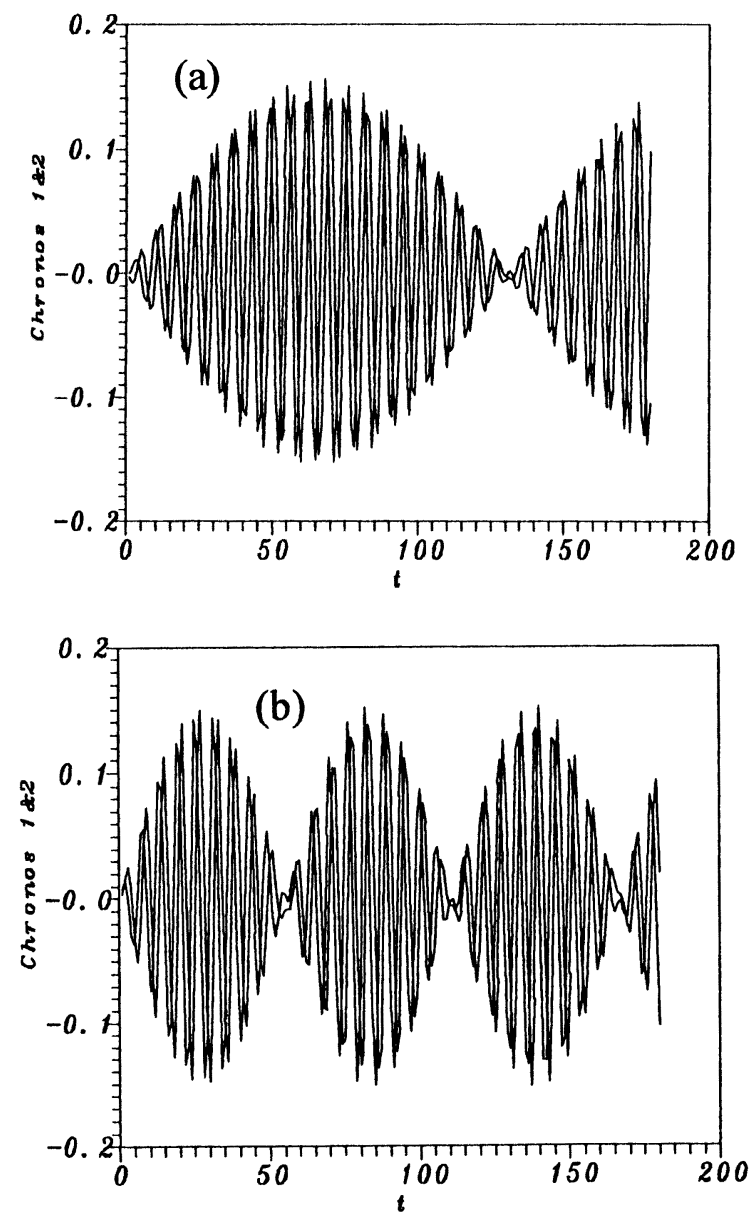

Fig. 6 Orthogonal temporal modes of the surface height ( a ) $\mathbf{U}^{\mathrm{a}}=0.95 \mathbf{c}(\mathbf{k})$ and ( b ) $\mathbf{U}^{\mathrm{a}}=1.1 \mathbf{c}(\mathbf{k})$ monic is resonantly generated. Out of these two conditions, B. O. D. shows that surface waves are of modulated temporal modes as it is seen in Fig. 6(a, b). In addition, evaluation of the entropy and the global energy of each space-time structure, i. e, a pair of $\left(\phi_{n}\right.$, $\boldsymbol{\psi}_{n}$ ) shows that the entire surface wave energy is contained only in the two first space-time structures. Eigenvalues $\alpha_{1}$ and $\alpha_{2}$ are large compared with others eigenvalues $\alpha_{n}(\mathbf{n} \geq 3)$. Therefore the truncated sequence $\eta(\mathrm{x}, \mathrm{t})=\sum_{\mathrm{n}=1}^{2} \alpha_{\mathrm{n}} \phi_{\mathrm{n}}(\mathrm{x}) \phi_{\mathrm{n}}(\mathrm{t})$ is a best approximation of the actual generated surface waves.

\section{The experiments}

The experiments were conducted in the $1 / 5$-scale model of the large I. R. P. H. E. wind-wave tank described in Houri ${ }^{17)}$. A key part of the investigation was the use of a device capable of generating perturbations with controllable dynamical and kinematical properties in the air flow. Within the context of the analysis presented above, the air-flow perturbations were designed to be a vortex street. The vortex generator developed by Giovanangeli \& Memponteil ${ }^{10)}$ in 1985 is used : it consists in a two-dimensional flapping airfoil of $10 \mathrm{~cm}$ chord placed above the upstream beach of the wind-wave tank. The airfoil (figure 7) oscillated between a horizontal position and a maximum angular displacement $\theta$ close to 30 degrees. With this vortex generator we made an extensive search to find a range of the vortex-generator frequency $\mathbf{n}_{0}$ and the wind velocity, $\mathbf{U}^{\mathrm{w}}$ controlled by the facility, to satisfy as far as possible the resonance conditions and the vortex street stability. A small domain of $\mathbf{n}_{0}$ and $\mathbf{U}^{\mathrm{w}}$ values, where resonance criteria seems possible to appear, is found. It was decided to run the experiment with frequency and wind velocity ranging from $1.5 \mathrm{~Hz}$ to 2 $\mathrm{Hz}$ and $0.50 \mathrm{~m} / \mathrm{s}$ to $2 \mathrm{~m} / \mathrm{s}$ respectively. The resonance condition could then be satisfied at wind velocity $\mathbf{U}^{\mathrm{w}}$ of order $0.90 \mathrm{~m} / \mathrm{s}$. An estimate of the vortex street convection velocity, $U^{\mathrm{a}}$, was made on the basis of the classical formula

$$
U^{\mathrm{a}}=\frac{2 \pi \Delta \mathrm{xn}_{0}}{\Phi\left(\mathrm{n}_{0}\right)}
$$

where $\mathbf{n}_{0}$ is the frequency, $\Delta \mathrm{x}$ the distance between two hot wires and $\boldsymbol{\Phi}\left(\mathbf{n}_{0}\right)$ the phase of the cross-spectral density of the signal from the two hot wires. The water -surface deflections were measured using capacitance wave gauges. The gauges and the hot wires were mounted on a movable carriage to record the variations at any desired fetch value.

Observations were made at fetches $\mathbf{x}_{1}=52 \mathrm{~cm}$ and $\mathbf{x}_{2}$ $=176 \mathrm{~cm}$. For the particular value $\mathbf{U}^{\mathrm{w}}=0.92 \mathrm{~m} / \mathrm{s}$ of the wind velocity, the air vortices advance with the measured phase celerity of the fundamental wave harmonic $\left(U^{\mathrm{a}}=\mathbf{c}_{m}\left(\mathbf{n}_{0}\right)=0.76 \mathrm{~m} / \mathrm{s}, n_{0}=2 \mathrm{~Hz}\right.$ ) which agrees quite well with $\mathbf{c}_{\mathrm{th}}\left(\mathbf{n}_{0}\right)=0.78 \mathrm{~m} / \mathrm{s}$, the theoretical fundamental wave speed. At this resonance criteria, using Fourier analysis it is shown, in agreement with the numerical 


\section{Wind $U^{w}(\mathrm{~m} / \mathrm{s})$}

\section{TROUEY}

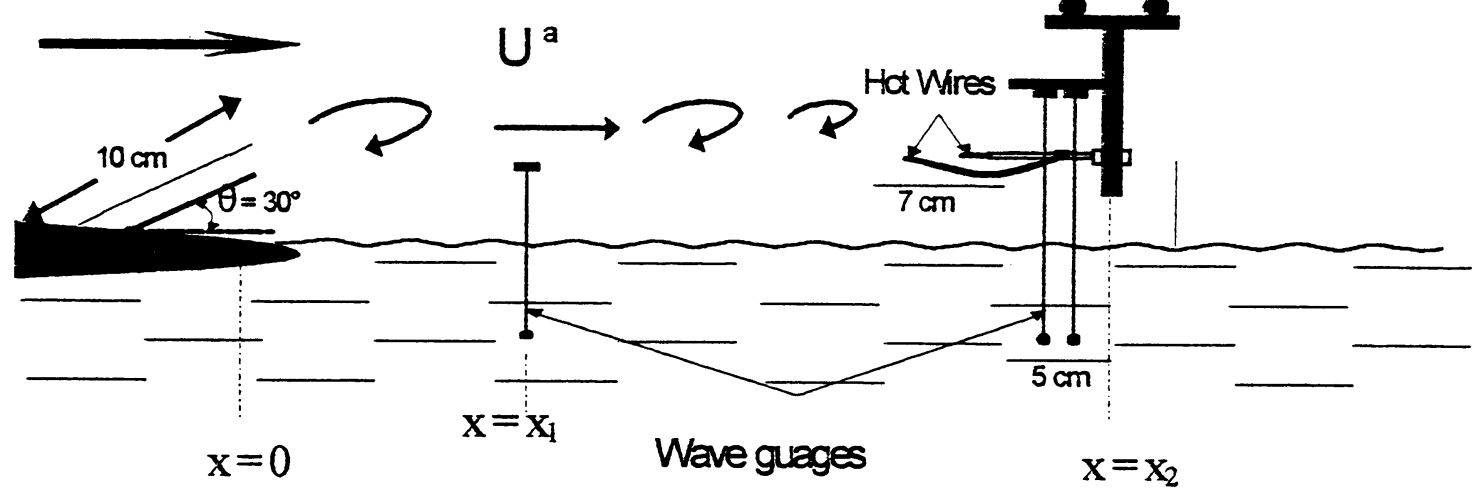

Fig. 7 Experimental setup
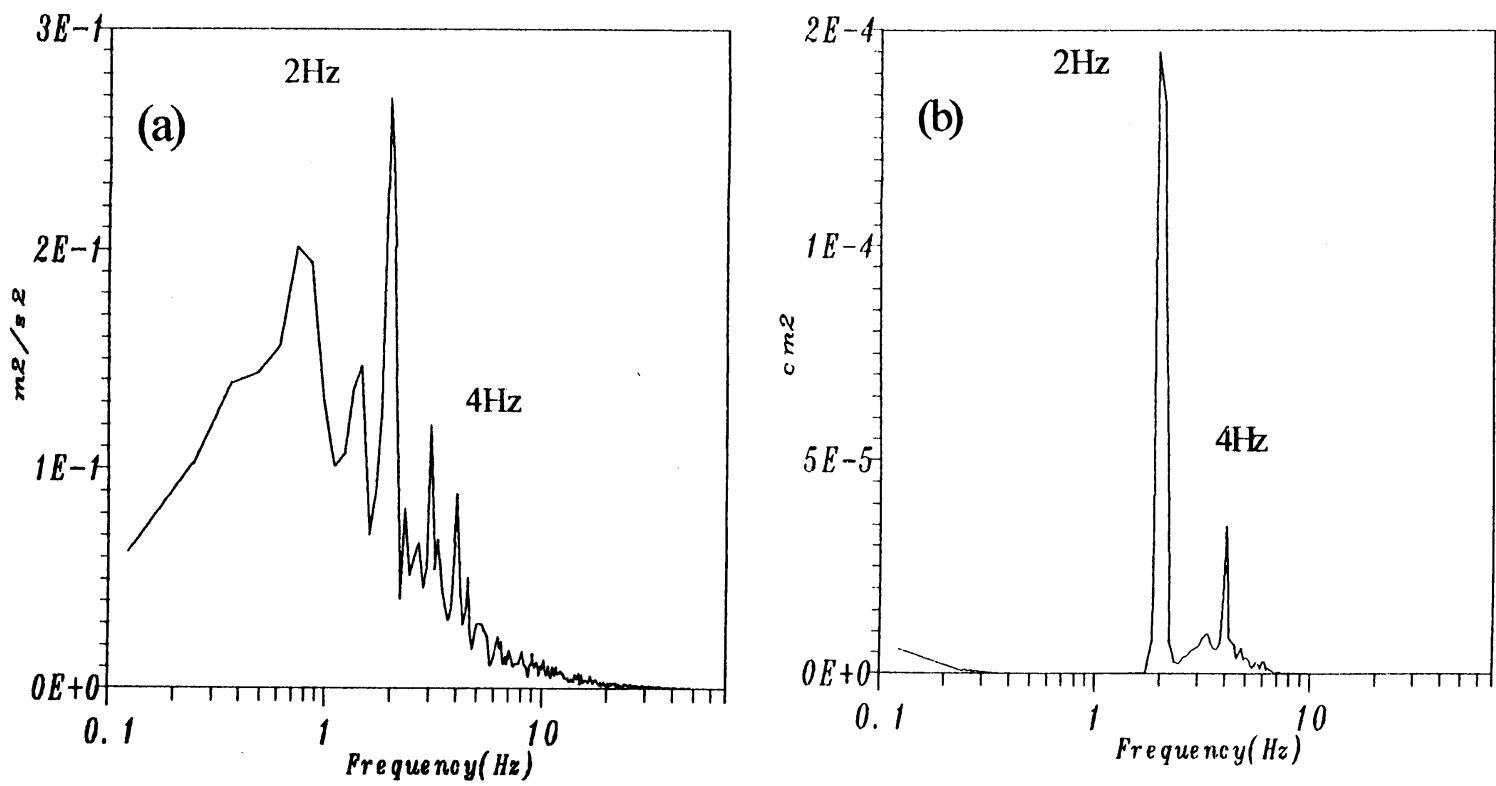

Fig. 8 Power spectral density of (a ) the longitudinal component of the air velocity fluctuations ( $b$ ) the water deflection level at the resonance of the fundamental harmonic.

prediction, that surface waves of basic frequency $\mathbf{n}_{0}$ are being resonantly generated as it is seen through power wind and wave spectral densities displayed in Fig. 8 (a, b) respectively. But Fourier analysis applied to the data obtained in the case of a vortex street moving at the measured phase speed of the second harmonic ( $U^{\mathrm{a}}$ $\left.=\mathbf{c}_{m}\left(2 \mathbf{n}_{0}\right)=0.60 \mathrm{~m} / \mathrm{s}, \quad \mathbf{n}_{0}=1.5 \mathrm{~Hz}, \quad \mathbf{c}_{\mathrm{th}}\left(2 \mathbf{n}_{0}\right)=0.52 \mathrm{~m} / \mathrm{s}\right)$ shows, contrary to Phillips theory (1957), that a peak of energy can occur at the second harmonic of the wave field with a peak of energy in the vortex field concentrated at the basic harmonic as it is seen in Fig. 9 (a, b).
However, wavelet analysis shows for the same data at a certain sharply localized moments, the existence of an appreciable energy at the second harmonic of the vortex field and that wave energy is effectively concentrated at the frequency of the second wave harmonic (Fig. 10). We suppose that this localized energy in the vortex field occurs sporadically and contributes with a strong manner to the generation of the second harmonic by means of resonance mechanism. The wavelet analysis used may prove useful for further explorations of transient couplings in wind-wave interactions. In a 

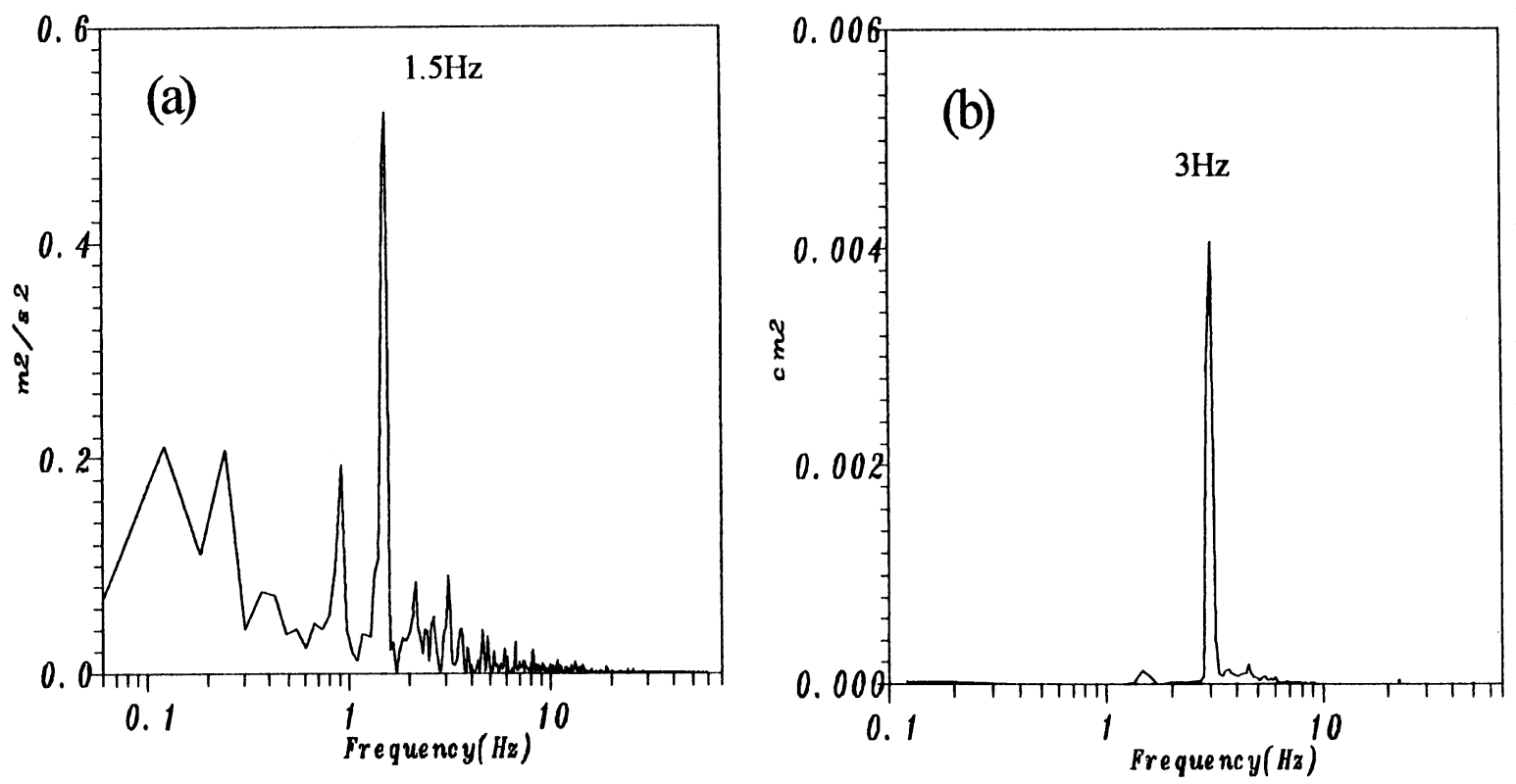

Fig. 9 Power spectral density of (a) the longitudinal component of the air velocity fluctuations ( $b$ ) the water deflection level at the resonance of the second harmonic
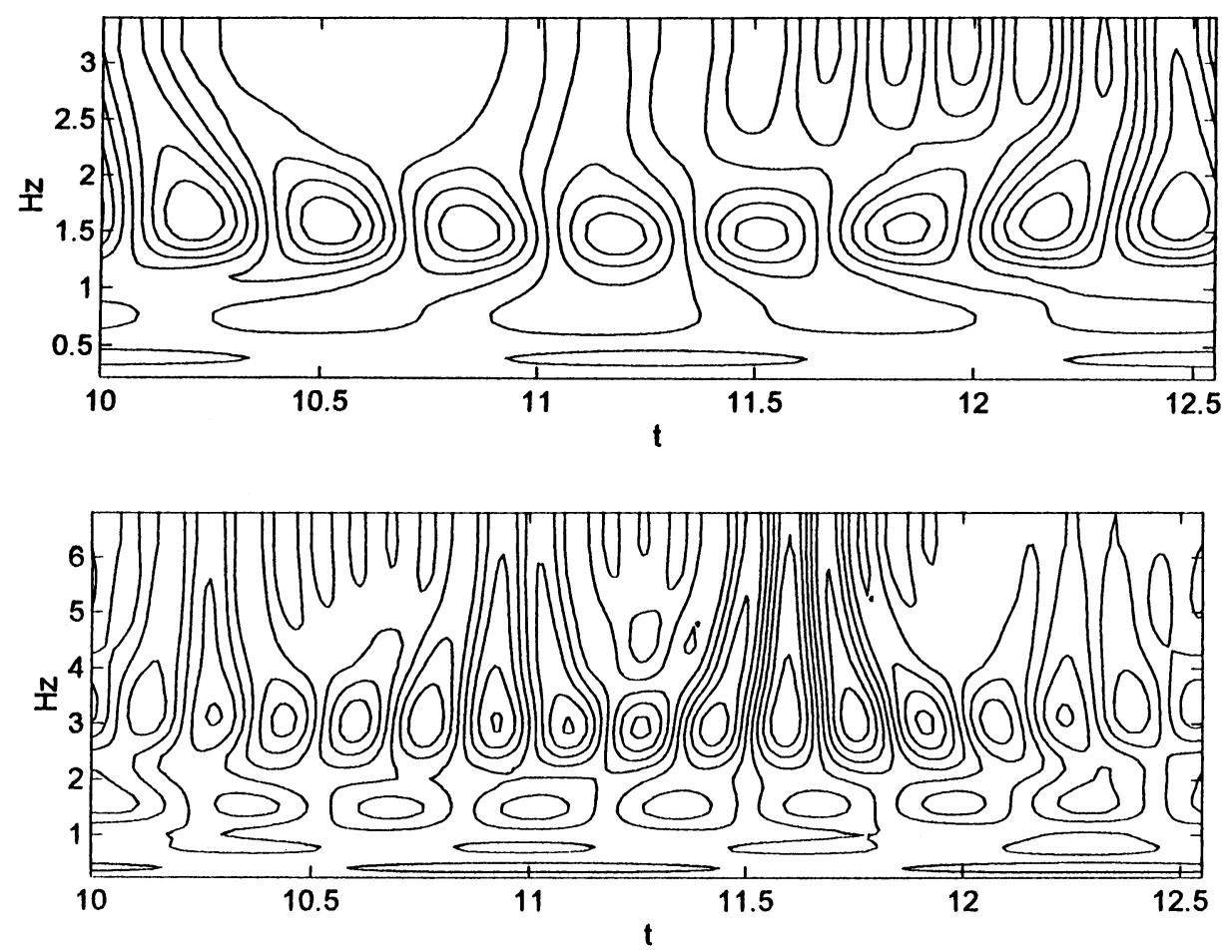

Fig. 10 The moduli of the wavelet transform of (upper) the air flow velocity fluctuations and (lower) the wave height 
private communication with Professor Phillips, he agreed with this additional result to its original theoretical work and confirm that our work demonstrates both experimentally and numerically that air pressure fluctuations can generate superharmonic wave component by means of a 'second harmonic resonance mechanism' additional to his 'first harmonic resonance mechanism' responsible for the growth of the basic wave component.

\section{Conclusions}

First we briefly summarize the main results of the paper and then outline some of their straightforward perspectives.

Exact nonlinear free surface governing equations are solved using the 'vortex sheet method'. A space-time analysis of the generated surface waves has been performed using the bi-orthogonal decomposition. It is found, depending on the convection speed of the air vortex street, that resonant excitation may occur at different harmonics of the fundamental frequency. Under the action of the induced vortex pressure, the basic wave or its harmonic can grow in time and their profiles become asymmetric characteristic of breaking wind-waves. The spatial characteristics of surface waves are found, through their orthogonal spatial modes, to be strongly related to the vortex street parameters. Particularly, for the wavelength of the resonant second harmonic which is half of the vortex wavelength. In order to observe the resonance process in experimental facilities, a vortex street is artificially created in a windwave tunnel. In agreement with the numerical formulation, it is found using, Fourier analysis, that both fundamental wave component and its second harmonic can be resonantly generated at suitable vortex street convection speed. Moreover, wavelet analysis pointed out that at a certain sharply localized moments, sporadic phenomenon may occur in the vortex field and may greatly contribute to the generation and the amplification of free surface wave's harmonic.

In another way, we can think about the bi-orthogonal decomposition as an efficient tool to predict and analyse a space-time bifurcation events, namely a qualitative change in the spatio-temporal nature of the surface wave (R. Lima $\left.{ }^{19)}\right)$. This will take place when two eigenvalues $\left(\boldsymbol{\alpha}_{n}\right)$ are degenerate in the biorthogonal spectrum (1). Such a scenario applied to the superposition of traveling waves leads to the generation of additional waves propagating at new celerities, which can be computed from spatial and temporal modes (chronos and topos) (see Houri ${ }^{17)}$ ).

Under given resonance conditions, bi-orthogonal decomposition and wavelet transform applied respectively to computed nonlinear surface waves and experimental data obtained in a wind-wave tunnel, suggest that coherent structures present in the atmospheric boundary layer of spatial periodicity $\mathbf{L}$ may generate surface waves of scales $\mathbf{L} / \mathbf{n}$ (superharmonic waves) or $\mathbf{n L}$ (subharmonic waves) if they move with a speed satisfying the dispersion relation of surface waves. Such a nature conceived with B. O. D. and/or W. T. analysis shall become a very useful tool to interpret sporadic phenomenon in many sides of wave tank experiment.

This paper was completed under the grants of the "Franco-Japanese" cooperation on Breaking Ocean Waves.

\section{References}

1) H. Lamb: Hydrodynamics. Cambridge University Press, 1932.

2) Phillips O.M.: On the generation of waves by turbulent wind. J. F. M., vol. 2, pp. 417-445, 1957.

3) Miles J. W.: On the generation of surface waves by shear flows. J. F. M., vol. 3, pp. 185-204, 1957.

4) Miles J. W. : On the generation of surface waves by shear flows. Part 2. J. F. M., vol. 6, p. 568, 1959.

5) Svendsen I. A. : Mixed boundary value problem for Laplace's equation. Coastal Engineering Laboratory Report. Technical University of Denmar$\mathrm{k}, 1971$.

6) Longuet-Higgins M.S. and Cokelet E. D: The deformation of steep surface waves on water: Numerical method of computation, Proc. Roy. Soc. Lond. A 350 1-26, 1976.

7) Vinje T. and Brevig P.: Numerical simulation of breaking waves. Adv Water Resources 4, pp. 77$82,1982$.

8) Zarodny and Greenberg: On a vortex sheet approach to the numerical Calculation of water waves, J. of Computational Physics vol. 11, pp. 440-446, 1973.

9) Baker G. R., Meiron D. I. and Orzag S. A. : Generalized vortex methods for free surface flow problems. J. Fluid Mech. 123, pp. 477-501, 1982.

10) Giovanangeli J. P. and Memponteil A. : Resonant and non-resonant waves excited by periodic vortices in airflow on water. J. Fluid Mech., vol. 159, pp. 69-84, 1985.

11) Novikov Y. A.: Generation of surface waves by discrete vortices, Izvestiya, 17, pp. 709-714, 1981.

12) Aubry N., Guyonnet R. and Lima R. : Spatiotemporal analysis of complex signals Theory and applications. J. Stat. Phys., 64, 683-739, 1991.

13) Houri M., Kharif C. and Giovanangeli J. P : Génération d'ondes à l'interface air-eau par une allée de tourbillons aériens mobiles. 11 ème Congrès Français de Mécanique, Lille, 1993 a.

14) Houri M., Kharif C. and Giovanangeli J. P: Generation of gravity waves by a vortex street in air. In "The Air-Sea Interface". M. A. Donelan, W. H. Hui and W. J. Plant, eds. University of Toronto press, Toronto, $1993 \mathrm{~b}$.

15) Rioul O. and Duhamel P. : Fast Algorithms for Discrete and Continuous Wavelet Transforms. IEEE Transactions Inf. Theory. Vol. 38, N 2, pp. 569-586.

16) J. Morlet, G. Arens, I. Fourgeau and D. Giard: Wave propagation and sampling theory. Geophysics 47, pp. 203-236, 1982.

17) Houri M.: Génération d'ondes résonnantes a l'interface air-eau par une allée de tourbillons 
aériens. Doctor thesis. University of Aix-Marseille II, N² 20795 75, 1995.

18) Houri M., Kharif C. and Giovanangeli J. P : An experimental study of wind-wave generation by discrete air vortices. In the proceedings of the Eur. Geophysical Soc., part II, C 337, 1994.

19) R. Lima: Private communication, CPT, Marseille. 1997. 